\title{
Graphite Intercalation Compounds Derived by Green Chemistry as Oxygen Reduction Reaction Catalysts
}

Jianchao Zhao ${ }^{1,2 \ddagger}$, Joseph H. Dumont ${ }^{1 \neq}$, Ulises Martinez ${ }^{1}$, Javier Macossay ${ }^{3}$, Kateryna Artyushkovad, Plamen Atanassov ${ }^{5}$, Gautam Gupta $^{*}$

${ }^{1}$ Los Alamos National Laboratory, Los Alamos, NM 87545, USA.

${ }^{2}$ Chemical Engineering Department, University of Louisville, Louisville, KY, USA.

${ }^{3}$ Chemistry Department, University of Texas Rio Grande Valley, Edinburg, TX, USA

${ }^{4}$ Chemical Engineering Department, University of New Mexico, Albuquerque, NM, USA

5 Chemical \& Biomolecular Engineering and National Fuel Cell Research Center, University of California, Irvine, CA, USA

*Corresponding author: gautam.gupta@louisville.edu 
Nomenclature of catalysts.

- Graphite: as received graphite.

- $\mathrm{GIC}\left(\mathrm{FeCl}_{3}\right)$ : Graphite Intercalated Compound with iron chloride.

- N-G/C: Graphite Intercalated Compound with iron chloride nitrogen-doped at high temperature under ammonia atmosphere.

- $\mathrm{GIC}\left(\mathrm{H}_{2} \mathrm{SO}_{4}\right)$ : Graphite Intercalated Compound with sulfuric acid.

- m-Graphite: Ball-milled graphite.

- $m-G / C-(N)$ : Ball-milled expanded graphite with $N$ precursors. Non heat-treated.

- $m-G / C-H T(N)$ : Ball-milled expanded graphite with $\mathrm{N}$ precursors and subsequently heat-treated at high temperature.

- m-G/C-HT(N-Fe): Ball-milled expanded graphite with $\mathrm{N}$ and Fe precursors and subsequently heat-treated at high temperature. 


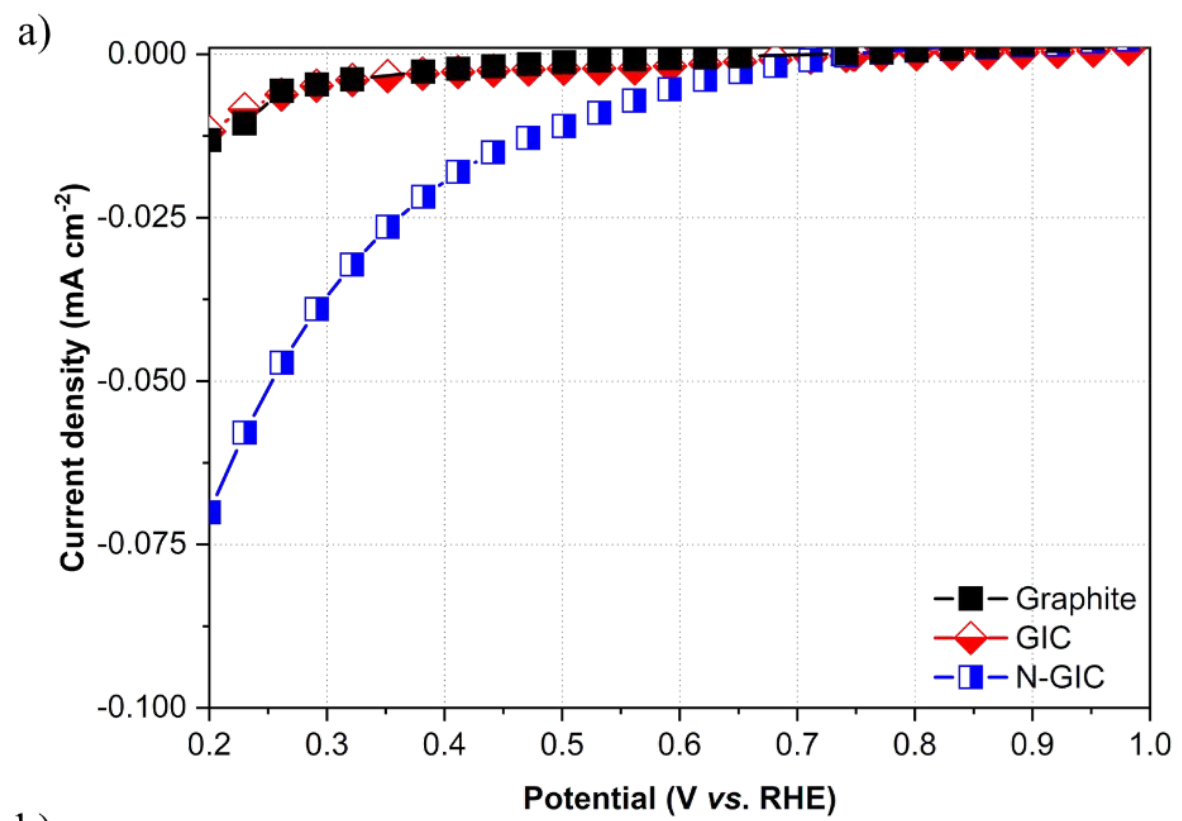

b)

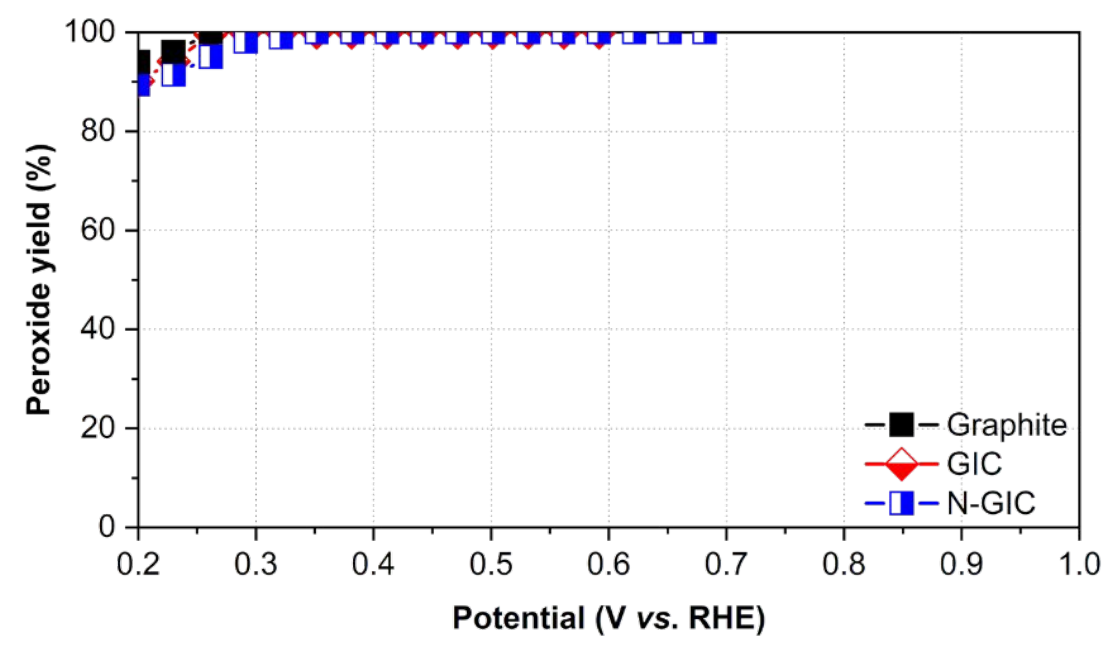

Figure S1. Electrocatalytic activity and selectivity of GIC catalysts for the oxygen reduction reaction. a) ORR activity in $0.5 \mathrm{M} \mathrm{H}_{2} \mathrm{SO}_{4}$, b) ORR selectivity in $0.5 \mathrm{M} \mathrm{H}_{2} \mathrm{SO}_{4}$; graphite data is added for comparison purposes. RRDE conditions: Steady-state 
voltammetry using $30 \mathrm{mV}$ potential step and $30 \mathrm{~s}$ potential hold time at every step; Pt ring held at a constant potential of $1.3 \mathrm{~V}$ vs. RHE; temperature $25^{\circ} \mathrm{C}$; rotation rate 900 r.p.m.

Supplementary table 1 | Comparison of the electrochemical performance of all materials in alkaline medium.

\begin{tabular}{|c|c|c|c|c|}
\hline \multirow[b]{2}{*}{ Catalyst } & \multicolumn{4}{|c|}{ Alkaline medium $(0.1 \mathrm{M} \mathrm{NaOH})$} \\
\hline & $\begin{array}{c}\text { E }_{\text {onset }} \\
\text { (V vs. RHE) }\end{array}$ & $\begin{array}{c}\mathrm{E}_{1 / 2} \\
\text { (V vs. RHE) }\end{array}$ & $\begin{array}{c}\text { Current } \\
\left(\mathrm{mA} \mathrm{cm}^{-2}\right) \text { at } 0.5 \mathrm{~V} \\
\text { vs. RHE }\end{array}$ & $\begin{array}{l}\mathrm{n} \text { at } 0.5 \mathrm{~V} \\
\text { vs. RHE }\end{array}$ \\
\hline Graphite & 0.7 & N/A & $N / A$ & 2 \\
\hline GIC & 0.73 & N/A & N/A & 2.5 \\
\hline N-GIC & 0.77 & N/A & N/A & 2.9 \\
\hline m-Graphite & 0.79 & N/A & N/A & 2.2 \\
\hline m-GIC-(N) & 0.82 & 0.73 & 1.7 & 2.2 \\
\hline m-GIC-HT(N) & 0.89 & 0.73 & 2.5 & 3.1 \\
\hline $\mathrm{m}-\mathrm{GIC}-\mathrm{HT}(\mathrm{N}-\mathrm{Fe})$ & 0.82 & 0.72 & 1.4 & 3 \\
\hline
\end{tabular}


Supplementary table 2 | Comparison of the electrochemical performance of all materials in acidic medium.

\begin{tabular}{|c|c|c|}
\hline & \multicolumn{2}{|c|}{ Acidic medium $\left(0.5 \mathrm{M} \mathrm{H}_{2} \mathrm{SO} 4\right)$} \\
\hline Catalyst & $\begin{array}{c}E_{\text {onset }} \\
\text { (V vs. RHE) }\end{array}$ & Peroxide yield (\%) \\
\hline Graphite & 0.4 & $<90 \%$ at $0.25 \mathrm{~V}$ vs. $\mathrm{RHE}$ \\
\hline GIC & 0.42 & $<90 \%$ at $0.25 \mathrm{~V}$ vs. $\mathrm{RHE}$ \\
\hline N-GIC & 0.7 & $<90 \%$ at $0.25 \mathrm{~V}$ vs. $\mathrm{RHE}$ \\
\hline m-Graphite & 0.42 & $15 \%$ at $0.5 \mathrm{~V}$ vs. RHE \\
\hline m-GIC-(N) & 0.52 & $40 \%$ at $0.5 \mathrm{~V}$ vs. RHE \\
\hline m-GIC-HT(N) & 0.82 & $42 \%$ at $0.5 \mathrm{~V}$ vs. RHE \\
\hline $\mathrm{m}-\mathrm{GIC}-\mathrm{HT}(\mathrm{N}-\mathrm{Fe})$ & 0.8 & $37 \%$ at $0.5 \mathrm{~V}$ vs. RHE \\
\hline
\end{tabular}




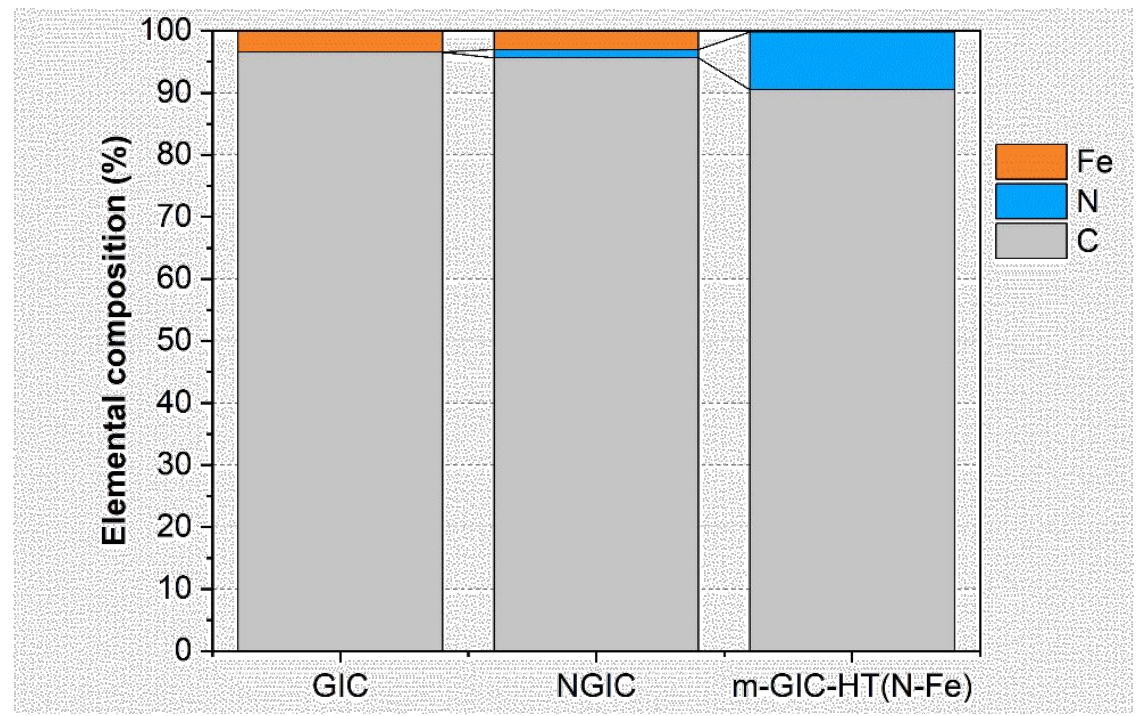

Figure S2. Elemental composition of graphite-based catalysts for the oxygen reduction reaction obtained by XPS. 

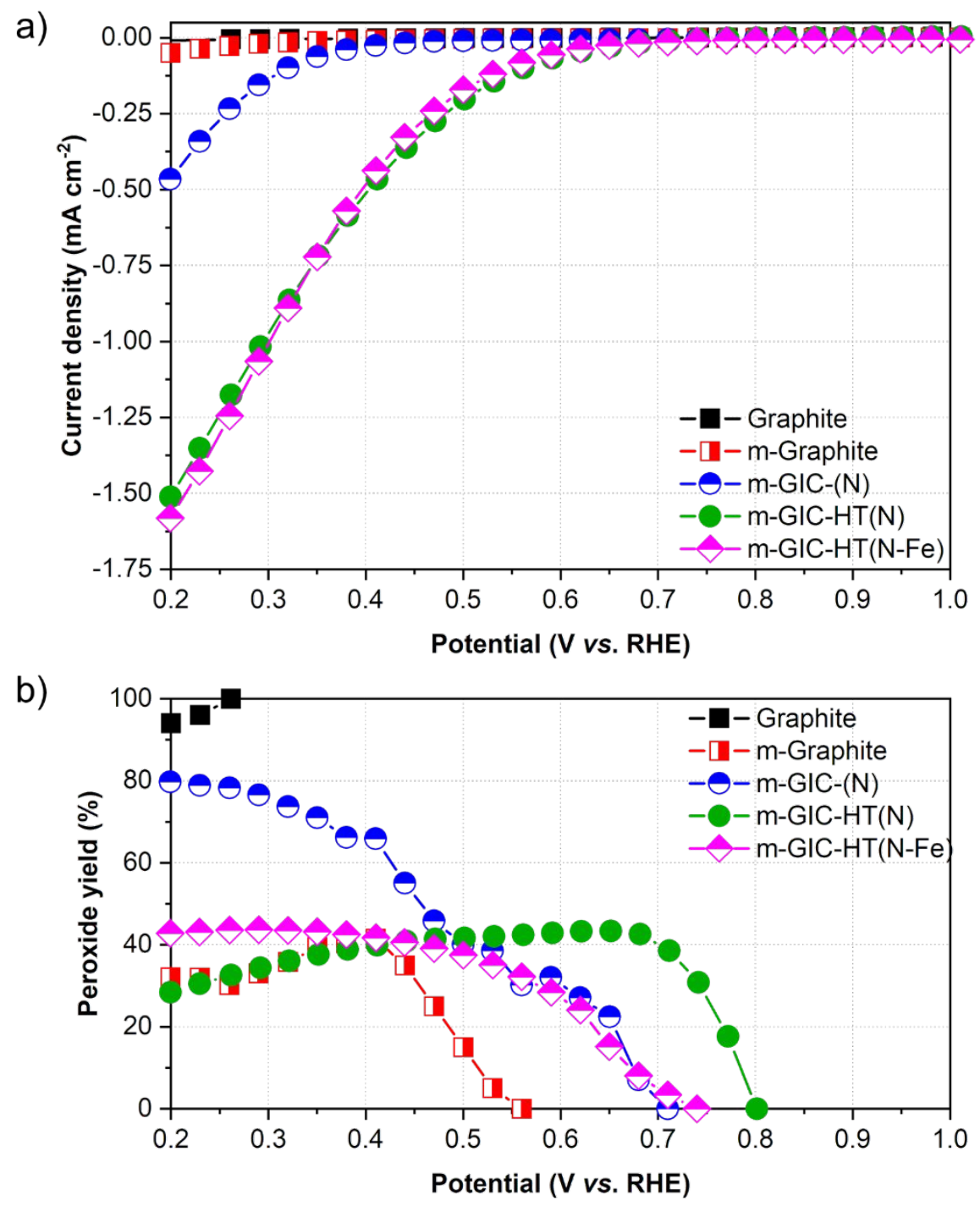

Figure S3. Electrocatalytic activity and selectivity of graphite and expanded and milled graphite-based catalysts for the ORR. a) ORR activity $0.5 \mathrm{M} \mathrm{H}_{2} \mathrm{SO}_{4}$. b) ORR selectivity in $0.5 \mathrm{M} \mathrm{H}_{2} \mathrm{SO}_{4}$; graphite data is added for comparison purposes. RRDE conditions: Steadystate voltammetry using $30 \mathrm{mV}$ potential step and $30 \mathrm{~s}$ potential hold time at every step; 
Pt ring held at a constant potential of $1.3 \mathrm{~V}$ vs. RHE; temperature $25^{\circ} \mathrm{C}$; rotation rate 900 r.p.m.

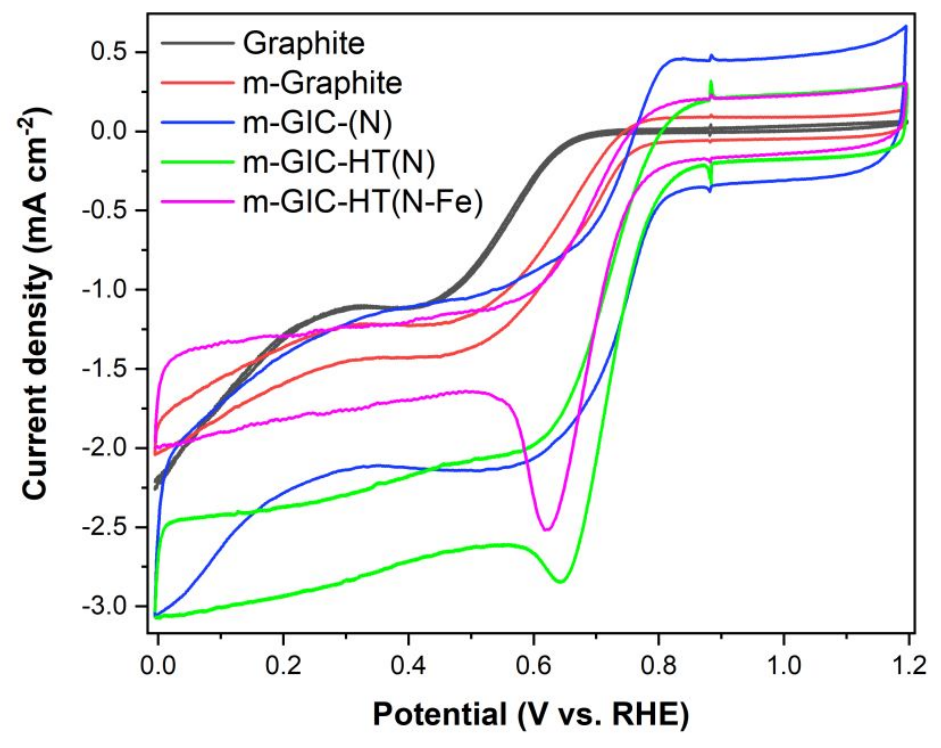

Figure S4. Cyclic voltammetry of graphite and expanded and milled graphite-based catalysts for the ORR. Graphite data is added for comparison purposes. RRDE conditions: Cyclic voltammetry at a rate of $50 \mathrm{mV} \mathrm{s}^{-1}$; temperature $25^{\circ} \mathrm{C}$; rotation rate 900 r.p.m. 